\title{
A CHARACTERIZATION OF ANALYTICITY. II
}

\section{KENNETH O. LELAND}

1. Introduction. We continue the study of families of analytic functions characterized by Lipschitz type conditions initiated in Analyticity. I [3]. A new property, $R$, is introduced and "smoothing" operators are developed, enabling the abstract theory to give as byproducts many results of the concrete theory, including a simplified proof of the analyticity of the elements of mean value families [2], and the solution of Neumann problem for the sphere in potential theory. Employing the smoothing operators another proof is obtained for the existence of derivatives in the finite dimensional case.

A family of functions $F$ on subsets of a linear space $B$ is called an $R$ family if $F$ is invariant under transformations of $B$ of the form $x \rightarrow r x, r>0$.

2. Notation and definitions. Let $B$ and $C$ be Banach spaces, and $F$ a family of continuous functions on open subsets of $B$ into $C$. Let $R$ denote the reals and $\omega$ the positive integers. $F$ is called a $T$ family if for $f, g \in F, r \in R, x \in B$, and $S$ an open set in $B, F$ contains $r f$, the function $f+g$ defined on $\operatorname{dom} f \cap \operatorname{dom} g$, the restriction $f \mid S$ of $f$ to $S$, and the translate $f_{x}$, where $f_{x}(y)=f(y-x)$ for $y \in\{x+t ; t \in \operatorname{dom} f\}$.

$F$ is called an $R$ family if for $f \in F, r>0, F$ contains the function $g$ such that $g(x)=f(r x)$ for $x \in\{t / r ; t \in \operatorname{dom} f\}$.

$F$ is called an $L$ family if $F$ is a $T$ family and if for all $\delta>0$, there exists $N(\delta)>0$, such that $f \in F, \quad M>0, \quad x \in B, \quad U_{x}(\delta)$ $=\{y \in B ;\|y-x\|<\delta\} \subseteq \operatorname{dom} f,\|f(y)\| \leqq M$ for $y \in U_{x}(\delta)$, implies

$$
\|f(y)-f(x)\| \leqq N(\delta) M\|y-x\| .
$$

If in (1) " $N(\delta)$ " is replaced by " $N / \delta$ " $F$ is called an $L_{N}$ family.

$F$ is said to be closed if for all sequences $f_{1}, f_{2}, \ldots$ in $F$ with a common domain $S$, which converge uniformly on $S$ to a limit function $f_{0}, f_{0} \in F$. For $\delta>0$, set $U(\delta)=U_{0}(\delta)$ and $U=U(1)$.

3. Property $R$. Property $R$ is possessed by $L_{N}$ families formed from complex analytic and harmonic functions. There are however important elementary examples which do not possess property $R$; in particular, the family of solutions of the equation " $\Delta f=c f, " c$ fixed, $c>0$, or $c<0$.

Received by the editors December 13, 1966. 
Let $B$ and $C$ be Banach spaces, $N, M>0, F$ an $L_{N}$ family from $B$ to $C$, and $f \in F$, such that $\operatorname{dom} f=B$, and $\|f(x)\| \leqq M$ for all $x \in B$. Then for $x \in B,\|f(x)-f(0)\| \leqq N M\|x\| \delta^{-1} \rightarrow 0$ as $\delta \rightarrow \infty, \delta>0$, and hence $\|f(x)-f(0)\|=0$, and $f$ is constant. Thus $F$ satisfies Liouville's theorem. Since clearly all $R L$ families are $L_{N}$ families, $N>0$, all $R L$ families must satisfy Liouville's theorem. Not all $L$ families satisfy Liouville's theorem, however. A trivial example is the $L$ family from $R$ to $R$ generated by the sine or cosine function.

We now proceed to the study of functions contained in an $R L$ family formed by differentiation and integration of elements of the family.

Theorem 1. Let $B$ and $C$ be Banach spaces, $n \in \omega, F$ a closed $T R$ family from $B$ to $C, \rho>0$, and $f \in F$, such that for $i=1, \cdots, n+1$, $f^{(i)}$ exists and is continuous. For $x \in U(\rho), i=1, \cdots, n$, set $h_{0}(x)$ $=f(0)$, and $h_{i}(x)=f_{0}^{(i)}(x, \cdots, x)$. Then $h_{i} \in F$ for $i=0,1, \cdots, n$. Moreover for some $\delta>0, F$ contains the function $\theta$, such that $\theta(x)=f_{x}^{\prime}(x)$ for $x \in U(\delta)$.

We observe from [3] that all $R L$ families satisfy the hypothesis of Theorem 1. We will need the case of $T R$ families in the study of mean value families in $\$ 5$.

Proof. There exists $\delta>0$ such that $U(\delta) \subseteq$ dom $f$. For $n \in \omega$, $x \in U(n \delta)$, set $g_{n}(x)=f(x / n)$. Then for $p>\rho / \delta, p \in \omega$, the sequence $g_{p}, g_{p+1}, \cdots$ converges uniformly on $U(\rho)$ to $h_{0}$.

There exists $\delta>0, M>0$, such that $U(3 \delta) \subseteq \operatorname{dom} f$ and $\left\|f_{x}^{\prime \prime}\right\| \leqq M$ for $x \in U(3 \delta)$. Let $x \in U(3 \delta)$ and for $t \in U(3 \delta)$, set $g(t)=f(t)-f_{x}^{\prime}(t-x)$. Then $g(x)=f(x)$ and for $t \in U(3 \delta)$,

$$
\begin{aligned}
\left\|g_{t}^{\prime}\right\|=\left\|f_{t}^{\prime}-f_{x}^{\prime}\right\| & \leqq\|t-x\| \sup \left\{\left\|f_{r}^{\prime \prime}\right\| ; r \in[t, x]\right\} \\
& \leqq\|t-x\| M
\end{aligned}
$$

and

$$
\begin{aligned}
\| f(t) & -f(x)-f_{x}^{\prime}(t-x) \| \\
& =\|g(t)-g(x)\| \\
& \leqq\|t-x\| \sup \left\{\left\|g_{r}^{\prime}\right\| ; r \in[t, x]\right\} \\
& \leqq\|t-x\|[\|t-x\| M]=M\|t-x\|^{2} .
\end{aligned}
$$

Let $|a| \leqq 2$, and for $x \in U(\delta), n \in \omega$, set $g_{n}(x)=n[f(a x+x / n)-f(a x)]$. Since $F$ is a $T R$ family, $g_{n} \in F$ for $n \in \omega$. Then for $x \in U(\delta), n \in \omega$, we have $a x+x / n, a x \in U(3 \delta)$, and 


$$
\begin{aligned}
\left\|g_{n}(x)-f_{a x}^{\prime}(x)\right\| & =n\left\|f(a x+x / n)-f(a x)-f_{a x}^{\prime}(x / n)\right\| \\
& \leqq n M\|x / n\|^{2}=M\|x\|^{2} / n .
\end{aligned}
$$

Thus the sequence $g_{1}, g_{2}, \cdots$ of $F$ converges uniformly on $U(\delta)$ to a limit function $w_{a}$, where $w_{a}(x)=f_{a x}^{\prime}(x)$ for $x \in U(\delta)$. Since $F$ is closed, $w_{a} \in F$. Let $r>\rho / \delta$. Then for $x \in U(\rho), \quad h_{1}(x)=f_{0}^{\prime}(x)=r f_{0}^{\prime}(x / r)$ $=r w_{0}(x / r)$, and $h_{1} \in F$. Set $\theta \equiv w_{1}$.

For $|a| \leqq 2, x \in U(\delta / 3), n \in \omega$, replacing $g_{n}(x)$ by $n\left[f_{a x+x / n}^{\prime}(x)-f_{a x}^{\prime}(x)\right]$ $=n\left[w_{a+1 / n}(x)-w_{a}(x)\right]$, and setting $w_{1, a}(x)=f_{a x}^{\prime \prime}(x, x)$, we obtain, when $a=0, h_{2} \in F$. Continuing this process the theorem follows.

Theorem 2. Let $B$ and $C$ be Banach spaces, $F$ a closed $R L$ family from $B$ to $C$, and $f$ a uniformly continuous function on $\bar{U}$ to $C$, such that $f(0)=0$, and $f \mid U \in F$. Then there exists a uniformly continuous function $g$ on $\bar{U}$ to $C$, such that $g \mid U \in F, g_{x}^{\prime}(x)=f(x)$ for all $x \in U$, and $\lim _{r \rightarrow 1}[g(x)-g(r x)] /(1-r)=f(x)$ for all $x \in \bar{U}-U$.

Proof. For $x \in \bar{U}, 0<r \leqq 1$, set $h_{r}(x)=f(r x) / r$, and set $h_{0}(x)=f_{0}^{\prime}(x)$. Let $\epsilon>0$. Then there exists $0<\rho<1$, such that for $x \in U(\rho)$, $\left\|f(x)-f(0)-f_{0}^{\prime}(x)\right\| \leqq \epsilon\|x\| / 2$. Then for $0<r, s \leqq \rho, x \in \bar{U}$, we have $r x$, $s x \in U(\rho)$, and

$$
\begin{aligned}
& \left\|h_{s}(x)-h_{r}(x)\right\|=\|f(s x) / s-f(r x) / r\| \\
& \quad \leqq s^{-1}\left\|f(s x)-f_{0}^{\prime}(s x)\right\|+r^{-1}\left\|f(r x)-f_{0}^{\prime}(r x)\right\| \\
& \quad \leqq s^{-1}[\epsilon\|s x\| / 2]+r^{-1}[\epsilon\|r x\| / 2] \\
& \quad=\epsilon / 2+\epsilon / 2=\epsilon .
\end{aligned}
$$

Since $f$ is uniformly continuous, there exists $0<\delta<\rho$, such that for $\rho \leqq r \leqq s \leqq 1,|s-r| \leqq \delta$, and $x \in \bar{U}$, we have $\left\|h_{s}(x)-h_{r}(x)\right\| \leqq \epsilon$.

For $x \in \bar{U}$, set $g(x)=\int_{0}^{1} h_{r}(x) d r,(1)$. For $x \in \bar{U}, n>1 / \delta, n \in \omega$,

$$
\begin{aligned}
\left\|g(x)-\sum_{0}^{n-1}(1 / n) h_{i / n}(x)\right\| & \leqq \sum_{0}^{n-1}\left\|\int_{i / n}^{(i+1) / n} h_{r}(x) d r-(1 / n) h_{i / n}(x)\right\| \\
& \leqq \sum_{0}^{n-1}\left\|\int_{i / n}^{(i+1) / n}\left[h_{r}(x)-h_{i / n}(x)\right] d r\right\| \\
& \leqq \sum_{0}^{n-1} \epsilon / n=n[\epsilon / n]=\epsilon,
\end{aligned}
$$

and thus $g \mid U$ is the limit on $U$ of the uniformly convergent sequence $\left\{\sum_{0}^{n-1}(1 / n) h_{i / n} \mid U ; n \in \omega\right\}$ in $F$. Since $F$ is closed, $g \mid U \in F$.

For $x \in U, x \neq 0$, and $n \in \omega, 1 / n<1-\|x\|$, substituting $s=r\|x\|$ in 
(1), we have $g(x)=\int_{0}\|x\| f(s x /\|x\|) s^{-1} d s$ and

$$
\begin{aligned}
g(x+x / n) & =\int_{0}^{\|x\|(1+1 / n)} f[s x(1+1 / n) /\|x+x / n\| \|] s^{-1} d s \\
& =\int_{0}^{\|x\|(1+1 / n)} f(s x /\|x\|) s^{-1} d s ;
\end{aligned}
$$

and thus

$$
\begin{aligned}
g_{x}^{\prime}(x) & =\lim _{n \rightarrow \infty} n[g(x+x / n)-g(x)] \\
& =\|x\| \lim _{n \rightarrow \infty}\|x / n\|-1 \cdot \int_{\left\|_{x}\right\|}^{\|x\|(1+1 / n)} f(s x /\|x\|) s^{-1} d s \\
& =\|x\|\left[f(\|x\| x /\|x\|)\|x\|^{-1}\right]=f(x) .
\end{aligned}
$$

REMARK. Theorem 2 may be used to solve the Neumann problem in potential theory for the sphere once the Dirichlet problem is solved for the sphere. Let $E$ be a Euclidean space, and let $\phi$ be a map (continuous function) of $H=\bar{U}-U$ into R, such that $\int_{H} \phi d \mu=0$, where $\mu$ is normalized surface measure on $H$. Let $f$ be a map on $\bar{U}$, such that $f \mid U$ is harmonic and $f \mid H=\phi$. Then $f(0)=\int_{H} f d \mu=\int_{H} \phi d \mu=0$. Since the family of harmonic functions on open subsets of $E$ into $R$ is an $R L$ family, from Theorem 2, there exists a map $g$ on $\bar{U}$, such that $g \mid U$ is harmonic, and

$$
\lim _{r \rightarrow 1}[g(x)-g(r x)] /(1-r)=f(x)=\phi(x) \quad \text { for } x \in H .
$$

4. Smoothing operators. The operators introduced in this section will enable us to approximate elements of a closed $T$ family $F$ by elements of $F$ which are as smooth, i.e., differentiable, as desired. They are similar to averaging operators used in potential theory [6] with the difference that here averages are taken over cubes rather than spheres.

Definition 1. Throughout the remainder of this paper $E$ shall denote a fixed Euclidean space, and $e_{1}, \cdots, e_{p}, p \in \omega$, a fixed orthonormal basis of $E$. Set $Q=\left\{x \in E ;-1 / 2 \leqq\left[x, e_{i}\right] \leqq 1 / 2, i=1, \cdots, p\right\}$ and for $i=1, \cdots, p$, set $Q_{i}=\left\{x \in Q ;\left[x, e_{i}\right]=0\right\}$.

Let $f$ be a map of an open set $S$ in $E$ into a Banach space $B$. Then for $x \in E, a>0$, such that $x+a Q=\{x+a y ; y \in Q\} \subseteq S$, set $L(f, a)(x)$ $=a^{-p} \int_{x+a Q} f(t) d m(t)$, where $m$ is Lebesgue measure on $E$.

ThForem 3. Let $B$ be a Banach space, $F$ a closed $T$ family from $E$ to 
$B, a>0$, and $f \in F$. Set $g=L(f, a)$ and let $H$ be a compact subset of the domain of $g$ with interior $S$. Then $g \mid S$ lies in $F$ and $g$ is continuously

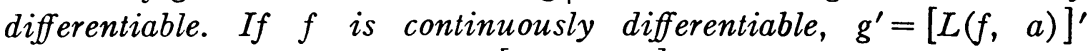
$=L\left(f^{\prime}, a\right)$. Thus $L(g, a)^{\prime \prime}$ and $L[L(g, a), a]^{\prime \prime \prime}$ exist and are continuous. Moreover, for $0<s<a, f_{s}=L(f, s)$ converges uniformly on $H$ to $f \mid H$ as $s \rightarrow 0$.

Proof. Let $\epsilon>0$ and set $M=H+a Q$. Then $M \subseteq \operatorname{dom} f$, and there exists $\delta>0$, such that $x, y \in M,\|y-x\|<\delta$, implies $\|f(y)-f(x)\|$ $\leqq \epsilon$. Let $0<r<\delta / p^{1 / 2}$, and $t_{1}, \cdots, t_{n} \in a Q, \quad n \in \omega$, such that $\left\{t_{i}+r Q ; i=1, \cdots, n\right\}$ is a subdivision of $a Q$. Then for $x \in H$, $i=1, \cdots, n, t \in t_{i}+r Q$, we have $x+t, x+t_{i} \in x+t_{i}+r Q \subseteq x+a Q$ $\subseteq H+a Q=M, \quad\left\|(x+t)-\left(x+t_{i}\right)\right\|=\left\|t-t_{i}\right\| \leqq p^{1 / 2} r<\delta, \quad$ and $\| f(x+t)$ $-f\left(x+t_{i}\right) \| \leqq \epsilon$. Thus for $x \in H$,

$$
\begin{aligned}
\left\|\sum_{1}^{n}(r / a)^{p} f_{-t_{i}}(x)-g(x)\right\| & \leqq a^{-p}\left\|\sum_{1}^{n} f\left(x+t_{i}\right) r^{p}-\int_{t_{i+r Q}} f(x+t) d m(t)\right\| \\
& \leqq a^{-p} \sum_{1}^{n}\left\|\int_{t_{i}+r Q}\left[f\left(x+t_{i}\right)-f(x+t)\right] d m(t)\right\| \\
& \leqq a^{-p} \sum_{1}^{n} \epsilon r^{p}=a^{-p}\left[\epsilon a^{p}\right]=\epsilon .
\end{aligned}
$$

Thus $g \mid S$ is the uniform limit on $S$ of elements of $F$ of the form $\sum_{1}^{n}(r / a)^{p} f_{-t_{i}}$. Since $F$ is closed, $g \mid S \in F$.

Similarly for $s>0, s>a, \delta$, and $x \in H$,

$$
\left\|f(x)-f_{s}(x)\right\|=s^{-p}\left\|\int_{s Q}[f(x)-f(x+t)] d m(t)\right\| \leqq s^{-p}\left[\epsilon s^{p}\right]=\epsilon,
$$

and thus $f_{s}$ converges uniformly on $H$ to $f$ as $s \rightarrow 0, s<a$.

Let $i=1, \cdots, p$, and set $\rho=a / 2$. Then $M_{i}=H+[-\rho, \rho] e_{i} \subseteq M_{i}$. For $x \in M_{i}$, set $g_{i}(x)=a^{-p} \int_{a Q_{i}} f(x+t) d m(t)$. Then for $x, y \in M_{i}$, $\|y-x\|<\delta$

$$
\begin{aligned}
\left\|g_{i}(y)-g_{i}(x)\right\| & =a^{-p}\left\|\int_{a Q_{i}}[f(y+t)-f(x+t)] d m(t)\right\| \\
& \leqq a^{-p}\left[\epsilon a^{p-1}\right]=\epsilon / a .
\end{aligned}
$$

For $x \in H, t \in E$, set $A_{x}(t)=\sum_{1}^{p}\left[t, e_{i}\right]\left[g_{i}\left(x+\rho e_{i}\right)-g_{i}\left(x-\rho e_{i}\right)\right]$. From (1), the function $A: x \rightarrow A_{x}(x \in H)$ is continuous. Then for $x \in S$, $t \in E$, such that $U_{x}(\|t\|) \subseteq S$, setting $s_{i}=\left[t, e_{i}\right]$ for $i=1, \cdots, p$, and setting $t_{1}=x$, and $t_{i}=x+\sum_{k=1}^{i-1} s_{k} e_{k}$ for $i=2, \cdots, p+1$, we have $t_{i} \in S$ for $i=1, \cdots, p+1$, and $\left\|s_{i} e_{i}\right\|<\delta$ for $i=1, \cdots, p$ and 


$$
\begin{aligned}
&\left\|g(x+t)-g(x)-A_{x}(t)\right\|=\left\|\sum_{1}^{p} g\left(t_{i+1}\right)-g\left(t_{i}\right)-A_{x}\left(t_{i+1}-t_{i}\right)\right\| \\
& \leqq \sum_{1}^{p}\left\|g\left(t_{i}+s_{i} e_{i}\right)-g\left(t_{i}\right)-A_{x}\left(s_{i} e_{i}\right)\right\| \\
& \leqq \sum_{1}^{p} \| \int_{-\rho+s_{i}}^{\rho+s_{i}} g_{i}\left(t_{i}+s e_{i}\right) d s-\int_{-\rho}^{\rho} g_{i}\left(t_{i}+s e_{i}\right) d s \\
& \leqq \sum_{1}^{p} \| \int_{\rho}^{\rho+s_{i}} g_{i}\left(g_{i}\left(x+\rho e_{i}\right)-g_{i}\left(x-\rho e_{i}\right) d s-s_{i} g_{i}\left(x+\rho e_{i}\right) \|\right. \\
&+\left\|\int_{-\rho}^{-\rho+s_{i}} g_{i}\left(t_{i}+s e_{i}\right) d s-s_{i} g_{i}\left(x-\rho e_{i}\right)\right\| \\
&= \sum_{1}^{p}\left\|\int_{\rho}^{\rho+s_{i}}\left[g_{i}\left(t_{i}+s e_{i}\right)-g_{i}\left(x+\rho e_{i}\right)\right] d s\right\| \\
&+\left\|\int_{-\rho}^{-\rho+s_{i}}\left[g_{i}\left(t+s e_{i}\right)-g_{i}\left(x-\rho e_{i}\right)\right] d s\right\| \\
& \leqq \sum_{1}^{p}\left|s_{i}\right|(\epsilon / a)+\left|s_{i}\right|(\epsilon / a) \leqq \sum_{1}^{p} 2\|t\| \epsilon / a=2 p a^{-1} \epsilon\|t\|,
\end{aligned}
$$

and thus $g_{x}^{\prime}$ exists and $g_{x}^{\prime}=A_{x}$, and $g$ is continuously differentiable.

Assume that $f$ is continuously differentiable and let $\epsilon>0$. Then there exists $\rho>0$, such that $x, y \in M,\|y-x\|<\rho$, implies $\| f(y)-f(x)$ $-f_{x}^{\prime}(y-x)\|\leqq \epsilon\| y-x \|$. Then for $x \in S, t \in U(\rho)$, such that $x+t \in S$,

$$
\begin{gathered}
\left\|g(x+t)-g(x)-L\left(f^{\prime}, a\right)_{x}(t)\right\| \\
=\left\|a^{-p} \int_{x+a Q}\left[f(t+s)-f(s)-f_{s}^{\prime}(t)\right] d m(t)\right\| \\
\leqq a^{-p}\left[\epsilon\|t\| a^{p}\right]=\epsilon\|t\|,
\end{gathered}
$$

and $g_{x}^{\prime}=L\left(f^{\prime}, a\right)_{x}$.

Remark. Let $B$ be a Banach space and $F$ an $L$ family from $E$ to $B$. Then Theorem 3 may be used to give another proof [3] of the existence of derivatives of elements of $F$. Let $f \in F$. For $n \in \omega$, set $f_{n}$ $=L\left(f, 1 / 2^{n}\right)$. Let $x \in \operatorname{dom} f, \delta>0$, such that $H=\bar{U}_{x}(\delta) \subseteq \operatorname{dom} f$. Then there exists $q \in \omega$, such that $n \geqq q, n \in \omega$, implies $H \subseteq \operatorname{dom} f_{q}$. From Theorem 3 , for $n \in \omega, f_{n}$ lies in $F$ and is differentiable, and the sequence $f_{\boldsymbol{q}}, f_{\boldsymbol{q}+\mathbf{1}}, \cdots$ converges uniformly on $H$ to $f$. From Theorem 
3.4 of $[3]$, for $t \in U_{x}(\delta), f_{\imath}^{\prime}$ exists, and the sequence $\left(f_{q}\right)_{\imath}^{\prime},\left(f_{q+1}\right)_{\imath}^{\prime}, \cdots$ converges to $f_{t}^{\prime}$.

An extension to the infinite dimensional case, when $E$ is replaced by an arbitrary Banach space, is possible but tedious.

A useful provider of examples is the following consequence of Theorem 3.

THEOREM 4. Let $B$ be a Banach space, and $F$ a closed $T$ family from $E$ to $B$, such that for $x \in E, \delta>0,\left\{f \mid \bar{U}_{x}(\delta) ; \bar{U}_{x}(\delta) \subseteq \operatorname{dom} f, f \in F\right\}$ is finite dimensional. Then $F$ is an L family.

Proof. Let $\delta>0$, set $H=\bar{U}(\delta)$, and set $G=\{L(f, a) \mid H ; a>0, f \in F$, $H \subseteq \operatorname{dom} L(f, a)\}$. Then $G$ is a finite dimensional linear space, and from Theorem 3, the elements of $G$ are continuously differentiable on $H$. Let $g_{1}, \cdots, g_{n}, n \in \omega$, be a basis of $G$ and $\operatorname{set} N=\sup \left\{\left\|\left(g_{i}\right)_{t}\right\| ; t \in H\right\}$. Then $N<\infty$, and for $i=1, \cdots, n, y \in H$,

$$
\left\|g_{i}(y)-g_{i}(0)\right\| \leqq\|y\| \sup \left\{\left\|\left(g_{i}\right)_{t}^{\prime}\right\| ; t \in[0, y]\right\} \leqq\|y\| N .
$$

For $f \in G$, there exists a unique sequence of numbers $L_{1}(f), \cdots$, $L_{n}(f)$, such that $f=\sum_{1}^{n} L_{i}(f) g_{i}$. Since $G$ is finite dimensional and $L_{1}, \cdots, L_{n}$ are linear $L=\sup \left\{\left\|L_{i}\right\| ; i=1, \cdots, n\right\}<\infty$. Thus for $f \in G, M>0$, such that $\|f(y)\| \leqq M$ for $y \in H$,

$$
\begin{aligned}
\|f(y)-f(0)\| & =\left\|\sum_{1}^{n} L_{i}(f)\left[g_{i}(y)-g_{i}(0)\right]\right\| \\
& \leqq \sum_{1}^{n}\left\|L_{i}(f)\right\| \cdot\left\|g_{i}(y)-g_{i}(0)\right\| \\
& \leqq \sum_{1}^{n}[L M] \cdot[N\|y\|]=N(\delta) M\|y\|,
\end{aligned}
$$

where $N(\delta)=n N L$.

Let $f$ be an element of $F$ such that $H \subseteq \operatorname{dom} f$. Then from Theorem $3, f \mid H$ is the uniform limit on $H$ of elements of $G$, and hence $f$ satisfies (1), and $F$ is an $L$ family.

REMARK. The argument for Theorem 4 is somewhat simplified if for $E$ and $B$ we take $R$, and for $G$ we take the family of antiderivatives (indefinite integrals) of elements of $F$. In this case [1] all elements of $F$ are linear combinations of expressions of the form $x^{p} e^{a x}$, where $p=0,1, \cdots$, and $a$ is arbitrary.

\section{Volume mean families.}

THEOREM 5. Let $\mu$ be a nonnegative Borel measure on $E$, with compact 
support $K$, such that $K$ is contained in no proper subspace of $E$, and $\mu(K)=1$. Let $F$ be the family of all maps $f$ of open sets in $E$ into $R$, such that for $x \in E, \delta>0$, if $x+\delta K \subseteq \operatorname{dom} f$,

$$
f(x)=\int_{K} f(x+\delta t) d \mu(t) .
$$

Then the elements of $F$ satisfy an elliptic partial differential equation and are analytic.

A proof of this result involving Fourier transforms and the notion of weak solutions of Laplace's equation is given by Friedman and Littman [2]. A considerably simpler argument is given here using the machinery developed in this paper.

Proof. Trivially $F$ is a closed $T R$ family. Set $G=\{L[L(L(f, a), a), a]$ $a>0, f \in F\}$. From Theorem $3, G$ is a $T R$ family, $g^{(3)}$ exists and is continuous for $g \in G$, and $f \in F, S \subseteq E, \bar{S}$ compact, $\bar{S} \subseteq \operatorname{dom} f$, implies $f \mid S$ lies in the closure $G_{0}$ of $G$.

Let $H$ be the space of all symmetric bilinear functionals on $E \times E$ into $R$. For $\theta \in H$, set $P(\theta)=\int_{K} \theta(t, t) d \mu(t)$. For $x, y, r, s \in E$, set $\theta[x, y](r, s)=[x, r][y, s]$, and set $I^{\prime}(x, y)=[x, y]^{\prime}=P(\theta[x, y])$. Since $\mu$ is not supported on any proper subspace of $E,[x, x]^{\prime}>0$ for all $x \in E, x \neq 0$. Thus $I^{\prime}$ is an inner product on $E$. Let $E^{\prime}$ denote the new Euclidean space $\left\{E, I^{\prime}\right\}$ determined by $I^{\prime}$.

Let $T$ be a unitary transformation (rotation) of $E^{\prime}$ in to itself. Then

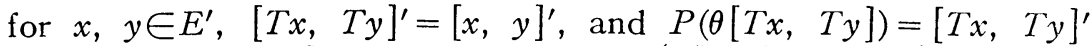
$=[x, y]^{\prime}=P(\theta[x, y])$. Now the collection $\{\theta[x, y] ; x, y \in E\}$ generates $H$. Thus with respect to $E^{\prime}, P$ is a rotation invariant operator operating on $H^{*}$, and hence letting $e_{1}^{\prime}, \cdots, e_{p}^{\prime}$ be an arbitrary orthonormal basis of $E^{\prime}$, there exists $c \neq 0$ such that $P(\theta)=c \sum_{1}^{p} \theta\left(e_{i}{ }^{\prime}, e_{i}{ }^{\prime}\right)$ for all $\theta \in H$.

Let $f \in G, x \in \operatorname{dom} f$, and $\rho>\sup \{\|t\| ; t \in K\}$, and set $h(t)=f_{x}^{\prime \prime}(t, t)$ for $t \in U(\rho)$. From Theorem 1, $h \in F$, and

$$
\begin{aligned}
c \sum_{1}^{p} \partial^{2} f(x) / \partial x_{i}^{\prime 2} & =c \sum_{1}^{p} f_{x}^{\prime \prime}\left(e_{i}^{\prime}, e_{i}^{\prime}\right)=P\left(f_{x}^{\prime \prime}\right)=\int_{K} f_{x}^{\prime \prime}(t, t) d \mu(t) \\
& =\int_{K} h(t) d \mu(t)=h(0)=f_{x}^{\prime \prime}(0,0)=0 .
\end{aligned}
$$

Thus the elements of $G$ are harmonic functions with respect to $E^{\prime}$. From [4], [5], the elements of $G_{0}$ and hence of $F$ are harmonic functions with respect to $E^{\prime}$, and hence analytic. 
REMARKs. Theorems 4 and 5 can also be handled from the standpoint of distribution theory [7]. We consider the family of functions $F$ in question as a family of distributions and take the closure $F_{0}$ of $F$ in the weak or distribution sense. In Theorem 5, employing property $R$, we observe that $F_{0}$ contains the functions $h_{x}(t)=f_{x}^{\prime}(t, t)$ which exist at least weakly. We then show that the elements of $F$ satisfy an elliptic partial differential equation at least weakly, and hence strongly.

In Theorem 4 [1], restricting attention to functions with real range, we have that the weak partial derivatives of the elements of $F$ lie in $F_{0}$. Since $F$ is finite dimensional, $F_{0}=F$, and hence the elements of $F$ are strongly differentiable.

The smoothing operators introduced in $\$ 4$ can be considered as analogues of convolutions of distributions with suitable approximate identity functions.

\section{REFERENCES}

1. P. M. Anselone and J. Korevaar, Translation incariant subspaces of finite dimension, Proc. Amer. Math. Soc. 15 (1964), 747-752.

2. A. Friedman and W. Littman, Functions satisfying the mean zalue property, Trans. Amer. Math. Soc. 102 (1962), 167-180.

3. K. O. Leland, A characierization of analyticity, Duke Math. J. 33 (1966), 551565 .

4. - A characterization of harmonic functions, (unpublished).

5. - Characterizations of solutions of " $\Delta f=c f$," Studia Math. 29 (to appear).

6. T. Rado, Subharmonic functions, Ergebnisse der Math., Vol. 5, Chelsea, New York, 1949.

7. K. Yosida, Functional analysis, Academic Press, New York, 1965.

UNIVERSITY OF VIRGINIA AND

ILLINOIS INSTITUTE OF TECHNOLOGY 